\title{
Increase in Iron and Zinc Concentrations in Rice Grains Via the Introduction of Barley Genes Involved in Phytosiderophore Synthesis
}

\author{
Hiroshi Masuda • Motofumi Suzuki • \\ Kendi Claudio Morikawa - Takanori Kobayashi • \\ Hiromi Nakanishi • Michiko Takahashi • \\ Masahiko Saigusa • Satoshi Mori • Naoko K. Nishizawa
}

Received: 29 February 2008 / Accepted: 3 July 2008 /Published online: 15 August 2008

(C) The Author(s) 2008

\begin{abstract}
Increasing the iron ( $\mathrm{Fe})$ and zinc $(\mathrm{Zn})$ concentrations of staple foods, such as rice, could solve Fe and $\mathrm{Zn}$ deficiencies, which are two of the most serious nutritional problems affecting humans. Mugineic acid family phytosiderophores (MAs) play a very important role in the uptake of $\mathrm{Fe}$ from the soil and $\mathrm{Fe}$ transport within the plant in graminaceous plants. To explore the possibility of MAs increasing the $\mathrm{Fe}$ concentration in grains, we cultivated three transgenic rice lines possessing barley genome fragments containing genes for MAs synthesis (i.e., $H v N A S 1$, $H v N A S 1$, and HvNAAT-A and HvNAAT-B or IDS3) in a paddy field with Andosol soils. Polished rice seeds with IDS3 inserts had up to 1.40 and 1.35 times higher Fe and $\mathrm{Zn}$ concentrations, respectively, compared to non-transgenic
\end{abstract}

H. Masuda, M. Suzuki, and K. C. Morikawa contributed equally to this work.

H. Masuda $\cdot$ M. Suzuki · T. Kobayashi $\cdot$ M. Takahashi $•$

N. K. Nishizawa $(\square)$

Department of Global Agricultural Sciences, Graduate School

of Agricultural and Life Sciences, The University of Tokyo,

1-1 Yayoi, Bunkyo-ku,

Tokyo 113-8657, Japan

e-mail: annaoko@mail.ecc.u-tokyo.ac.jp

K. C. Morikawa $\cdot$ M. Saigusa

Field Science Center, Tohoku University,

232-3 Osaki, Naruko Onsen,

Yomogida 989-6711, Japan

H. Nakanishi $\cdot$ S. Mori

Department of Applied Biological Chemistry, Graduate School

of Agricultural and Life Sciences, The University of Tokyo,

1-1 Yayoi, Bunkyo-ku,

Tokyo 113-8657, Japan rice seeds. Enhanced MAs production due to the introduced barley genes is suggested to be effective for increasing $\mathrm{Fe}$ and $\mathrm{Zn}$ concentrations in rice grains.

Keywords Biofortification · Iron deficiency · Mugineic acid family phytosiderophores $\cdot$ Transgenic rice $\cdot$ Zinc deficiency

\section{Introduction}

Iron $(\mathrm{Fe})$ and zinc $(\mathrm{Zn})$ are essential micronutrients in plants and animals. Fe deficiency is one of the most prevalent micronutrient deficiencies in the world, affecting an estimated two billion people [19] and contributing to 0.8 million deaths per year worldwide [28]. Zn deficiency is a serious nutritional problem, affecting an estimated one third of the world's population [18] and contributing to 0.8 million deaths per year. Fe and $\mathrm{Zn}$ deficiencies are the sixth and fifth highest health risk factors, respectively, in developing countries, and they have high mortality rates [28].

There are three main ways to solve micronutrient deficiencies: micronutrient supplementation, food fortification, and biofortification. Among these options, biofortification (i.e., the breeding of micronutrient-fortified crops) has great advantages because specific processing after harvest and special infrastructure are not required [13]. Therefore, this solution is suitable to all situations, especially for people who live in rural areas. In particular, using the transgenic approaches, it is possible to produce micronutrient-fortified rice varieties.

Fe fortification using a transgenic approach has been attempted using ferritin, a protein that stores approximately 
4,500 $\mathrm{Fe}$ atoms in a complex. Goto et al. [5] generated transgenic rice plants that expressed soybean ferritin (SoyferH1) in the endosperm using the endosperm-specific glutelin gene $(G l u B 1)$ promoter of rice; the transformants accumulated up to three times as much $\mathrm{Fe}$ in brown seeds. Furthermore, $\mathrm{Qu}$ et al. [17] generated rice transformants that expressed ferritin at different levels in seeds; the $\mathrm{Fe}$ concentration in brown seeds did not correspond with the enhanced ferritin expression level. Thus, the enhancement of ferritin expression may not be sufficient to further increase the Fe concentration in rice grains. Qu et al. [17] proposed that the enhancement of $\mathrm{Fe}$ uptake from the soil and translocation inside the plant, in addition to $\mathrm{Fe}$ storage in seeds, is important for further Fe fortification in seeds.

Graminaceous plants secrete mugineic acid family phytosiderophores (MAs) into the rhizosphere for $\mathrm{Fe}$ uptake from soil [14, 23]. MAs form Fe(III)-MAs complexes that are absorbed into the root via YS1 transporters [4]. Among the graminaceous plants, barley is highly tolerant to Fe deficiency and possesses a series of MAs biosynthetic genes, including NAS, NAAT, DMAS, IDS2, and $I D S 3$ ([2, 6, 11, 16, 25]; Fig. 1). In contrast, rice lacks the two dioxygenase genes (IDS2 and IDS3) and secretes only 2'-deoxymugineic acid (DMA; [16]). This is thought to be one reason why barley has a higher tolerance to $\mathrm{Fe}-$ deficiency than rice [11]. The expression of MAs biosynthetic genes such as barley HvNAS1, HvNAAT-A, $H v N A A T-B, H v D M A S 1, I D S 2$, and IDS3, is up-regulated in Fe-deficient barley roots [2, 6, 16, 25].

Higuchi et al. [8] produced transgenic rice lines that contained the barley $H v N A S 1$ gene. These rice lines expressed HvNAS1 not only in Fe-deficient roots and leaves but also in roots with sufficient Fe. Takahashi et al. [24] introduced a barley genome fragment containing HvNAAT$A$ and $H v N A A T-B$ into rice. The transformants exhibited increased Fe-deficiency tolerance and grew well in alkaline soil. Kobayashi et al. [11] introduced the barley IDS3 genome fragment into rice; the transformants secreted mugineic acid, a hydroxylated form of DMA (Fig. 1). Because these transformants contained introduced barley genome fragments, the expression of the genes encoding MAs synthesis was regulated by their own promoters, and the expression of the introduced genes was induced in $\mathrm{Fe}$ deficient roots and leaves $[8,11,24]$. Thus, these genes are expected to be expressed when and where the $\mathrm{Fe}$ requirement is elevated. This hypothesis suggests the possibility of enhancing $\mathrm{Fe}$ uptake and translocation for Fe fortification in the seed by introducing barley HvNAS1, $H v N A A T-A, H v N A A T-B$, and IDS3 into the rice genome.

Transgenic lines evaluated under greenhouse condition need to be evaluated under field condition. Therefore, we cultivated transgenic rice lines containing the introduced $H v N A S 1$ (gNAS1), HvNAS1, and HvNAAT-A and HvNAAT$B$ (gNAS1+gNAAT), or IDS3 (gIDS3) genes (Fig. 2) in a field with Andosol soils, which are highly porous, darkcolored soils developed from parent material of volcanic origin and one of the typical soil groups in Japan. The $\mathrm{Fe}$ and $\mathrm{Zn}$ concentrations in seeds were higher in gIDS3 than in the other transgenic and non-transgenic lines. These results suggest that the enhanced expression of MAs biosynthetic genes produces rice seeds with higher $\mathrm{Fe}$ and Zn content.
Fig. 1 The biosynthetic pathway of mugineic acid family phytosiderophores (MAS) in graminaceous plants. $S A M$ $S$-Adenosyl-methionine, $N A$ nicotianamine, DMA

2'-deoxymugineic acid, $M A$ mugineic acid, $H M A$

3-hydroxymugineic acid, epiHDMA 3-epihydroxy-2'deoxymugineic acid, epiHMA 3-epihydroxymugineic acid, SAMS S-adenosyl-methionine synthetase, NAS nicotianamine synthase, NAAT nicotianamine aminotransferase, DMAS 2'deoxymugineic acid synthase, IDS2 dioxygenase catalyzing the hydroxylation of three positions of DMA and MA, IDS3 dioxygenase catalyzing the hydroxylation of 2'-positions of DMA and epiHDMA

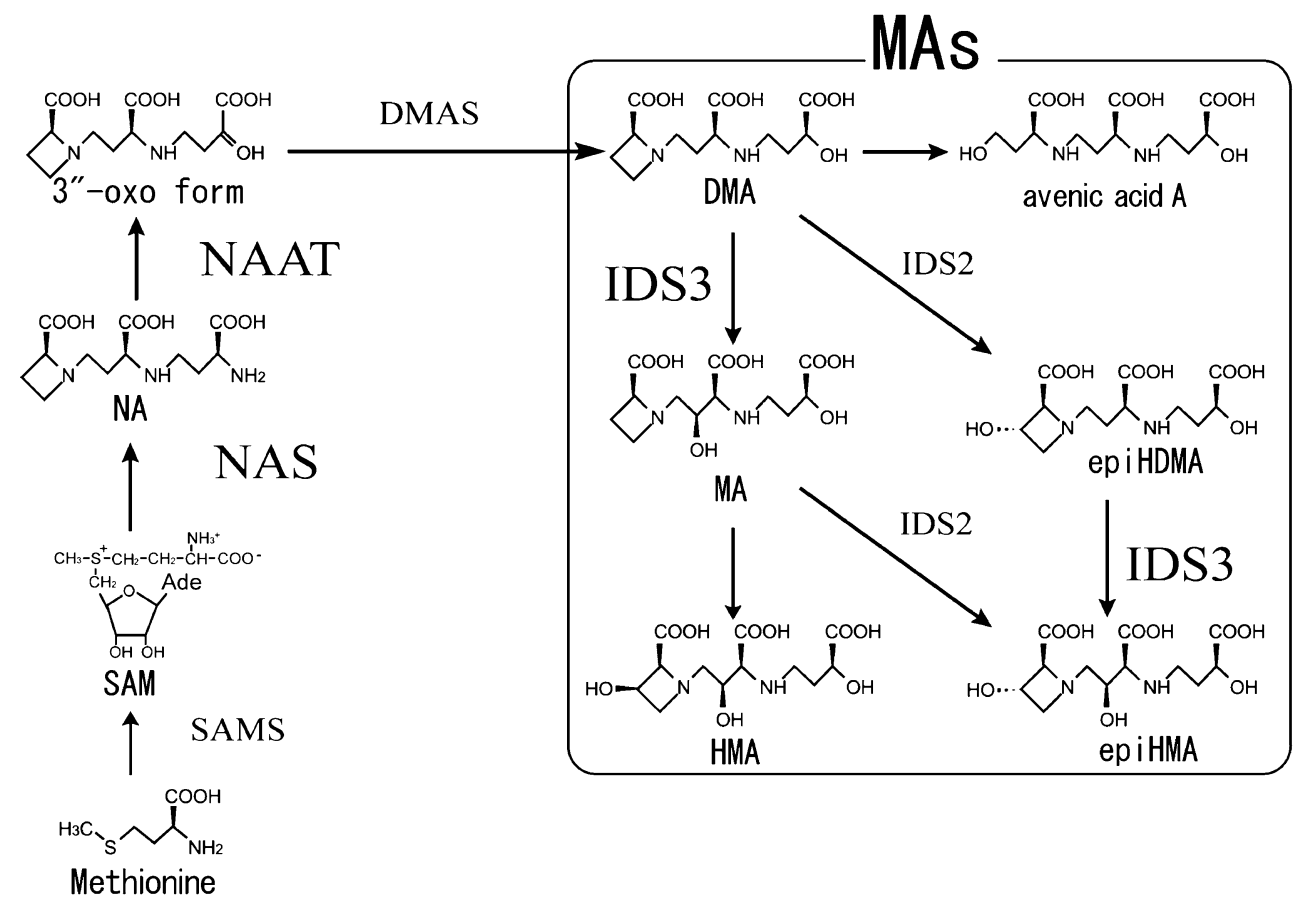




\section{(a) gNAS1}

\section{-}

\section{(b) gNAS1+gNAAT}

\section{-7[NPP]}

\section{(c) $\mathrm{gIDS} 3$}

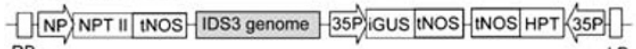

Fig. 2 T-DNA regions of the constructs introduced into three transgenic rice lines. a gNAS1: transgenic rice line with a barley 13.0-kb HvNAS1 genome fragment [8]. b gNAS1+gNAAT: transgenic rice line with barley $7.6-\mathrm{kb} H v N A S 1$ and $11.0-\mathrm{kb} H v N A A T-A$ and $H v N A A T-B$ genome fragments. c gIDS3: transgenic rice line with a barley $20-\mathrm{kb} I D S 3$ genome fragment [11]. $R B$ Right border, $L B$ left border, NP nopaline synthase promoter, NPT II neomycin phosphotransferase gene, $t N O S$ nopaline synthase terminator, $35 P$ cauliflower mosaic virus $35 \mathrm{~S}$ promoter, $i G U S \beta$-glucuronidase gene with an intron, $H P T$ hygromycin phosphotransferase gene

\section{Results}

Plant growth and grain yield

Three transgenic (gNAS1, gNAS1+gNAAT, and gIDS3) and one non-transgenic (NT) rice lines were used (Fig. 2). Each line was planted in four replications in an experimental field with Andosol soils (Fig. 3). During all growth stages, there were only slight differences between the NT, gNAS1, gNAS1+gNAAT, and gIDS3 plants in height, leaf color, and number of tillers (data not shown). At the heading stage on 97 days after transplanting (DAT), there were no significant differences in these indices between NT plants and transgenic rice plants (Fig. 4). There were no

a

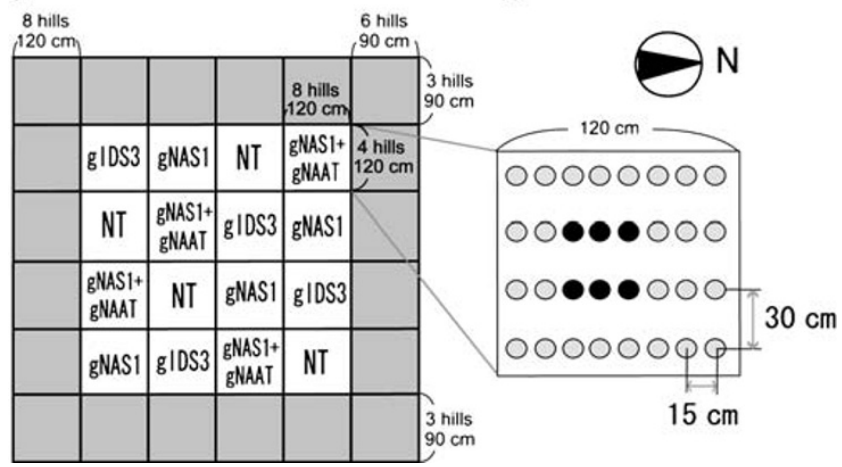

Fig. 3 Experimental design in the paddy field. a Replication placement in the paddy field. NT Non-transgenic plants, gNAS1 gNAS1 plants, $g N A S 1+g N A A T$ gNAS1+gNAAT plants, $g I D S 3$ gIDS3 plants. NT plants were also planted in the gray area to maintain uniform light and nutrient conditions. b Design of the hill settings in each replication. Each replication consisted of eight $(120 \mathrm{~cm}) \times$ four $(120 \mathrm{~cm})$ hills. The inner six hills, shown in black, were used for detailed analyses
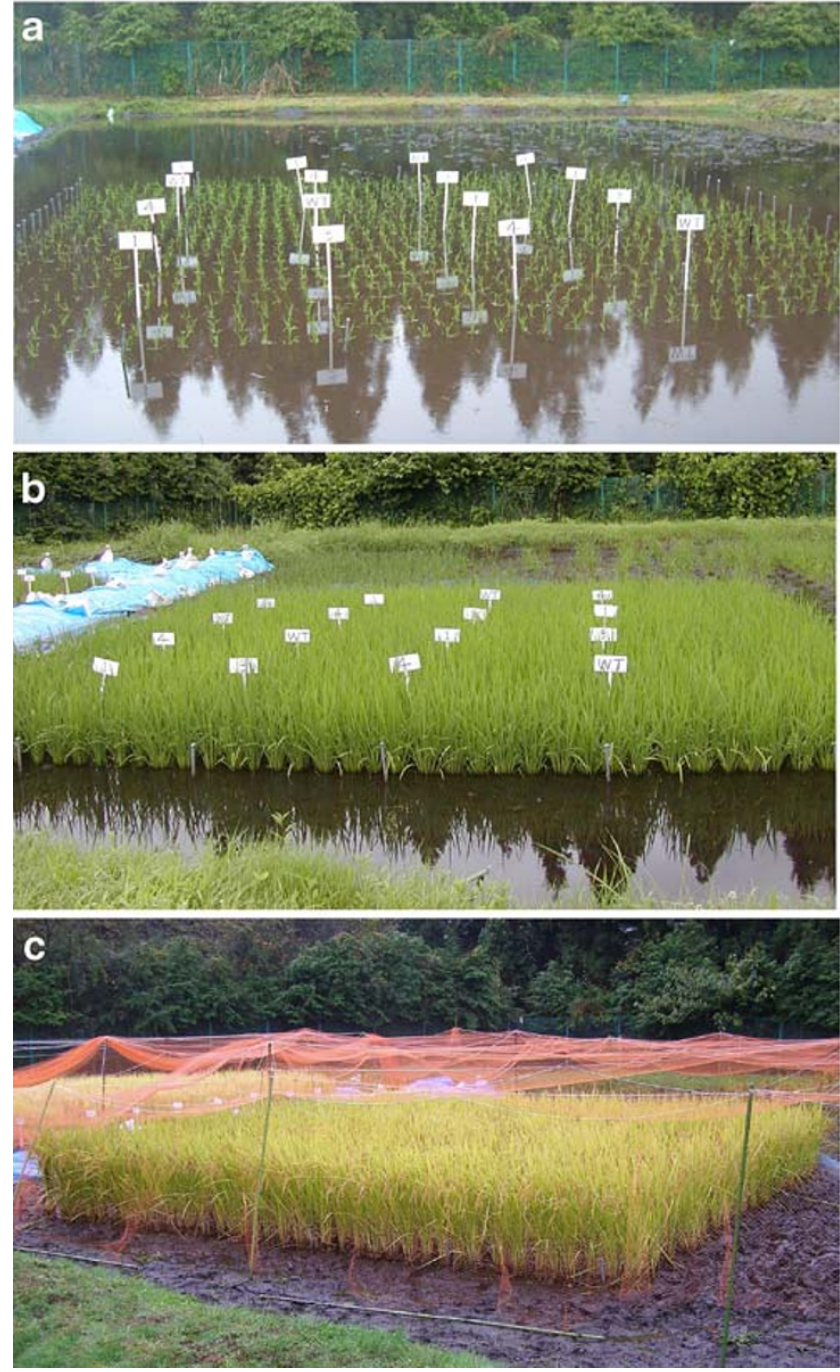

Fig. 4 Pictures of transgenic and non-transgenic plants in the experimental field with Andosol soils. Experimental design is illustrated in Fig. 3, photographed from west direction. WT Nontransgenic, 1 gNAS1 line, 3 gIDS3 line, 4 gNAS1-gNAAT line. a 2 days after transplanting (DAT), b 59 DAT and $\mathbf{c} 158$ DAT (harvested day)

significant differences between the NT and transgenic lines in grain yield (Fig. 5a), the number of harvested seeds (Fig. 5b), or the number of ears per hill and length of ears (data not shown).

Metal concentrations in brown and polished rice grains

The average Fe concentration in brown rice grains from the NT, gNAS1, gNAS1+gNAAT, and gIDS3 plants was 6.50, 5.98, 7.03, and $8.10 \mu \mathrm{g} \mathrm{g}^{-1}$, respectively (Fig. 6a). The average $\mathrm{Zn}$ concentration in brown rice grains from the NT, gNAS1, gNAS1+gNAAT, and gIDS3 plants was 15.0, 15.2, 16.1, and $19.4 \mu \mathrm{g} \mathrm{g}^{-1}$, respectively (Fig. 6b). In brown rice grains from gIDS3 plants, the $\mathrm{Fe}$ and $\mathrm{Zn}$ 
a

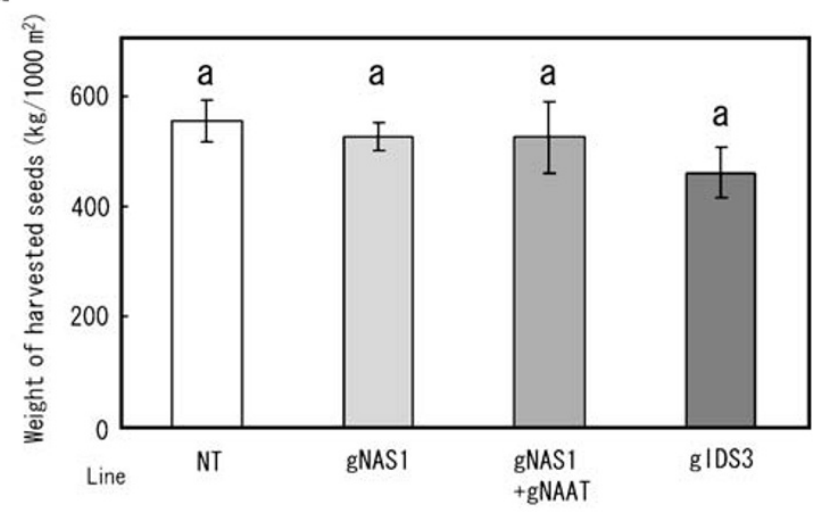

b

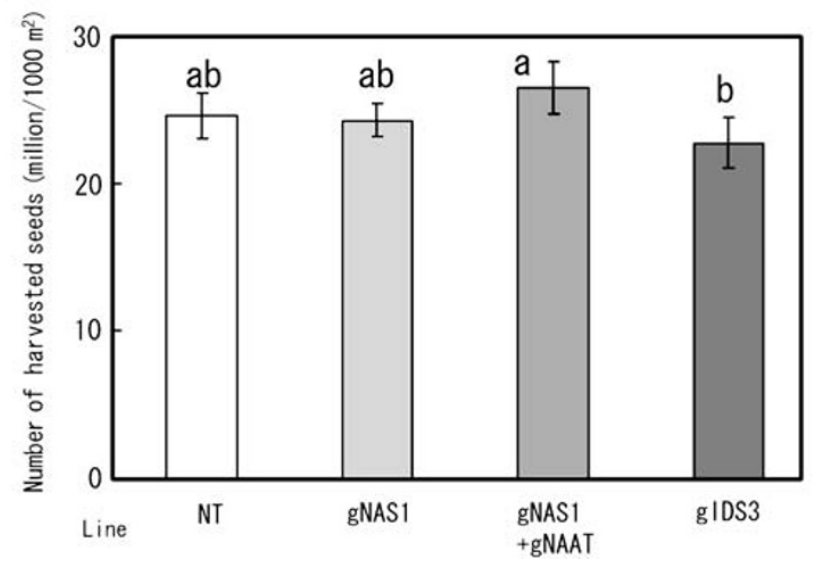

Fig. 5 Yields of the non-transgenic (NT), gNAS1, gNAS1 + gNAAT, and gIDS3 lines. a Weight of harvested seeds $\left(\mathrm{kg} / 1,000 \mathrm{~m}^{2}\right)$. b Number of harvested seeds (million seeds $/ 1,000 \mathrm{~m}^{2}$ ). The weight and number of seeds were measured for the inner six hills of each replication. The data represent the mean \pm standard error of four replications for each line. Seed weights were recalculated as containing $15 \%$ water. Different letters above the bars indicate significant differences

concentrations were 1.25 and 1.30 times those from NT plants, respectively. There were no significant differences between the transgenic and NT plants in the copper $(\mathrm{Cu})$ concentration in the grains (Fig. 6d). There was no significant difference between NT and gNAS1+gNAAT or gIDS3 plants in manganese $(\mathrm{Mn})$ concentration in the grains (Fig. 6c). In gNAS1 plants, the Mn concentration in the grains was somewhat lower than that in NT plants.

In polished rice grains, the average Fe concentration of NT, gNAS1, gNAS1+gNAAT, and gIDS3 plants was 1.06 , $1.11,1.19$, and $1.49 \mu \mathrm{g} \mathrm{g}^{-1}$, respectively (Fig. 7a). The average $\mathrm{Zn}$ concentration of NT, gNAS1, gNAS1 + gNAAT, and gIDS3 polished grains was 10.6, 11.3, 11.9, and $14.3 \mu \mathrm{g} \mathrm{g}^{-1}$, respectively (Fig. 7b). In polished rice grains from gIDS3 plants, the Fe and $\mathrm{Zn}$ concentrations were 1.40 and 1.35 times those from NT plants, respectively (Fig. 7a,b).
There were no significant differences between NT and transgenic lines in $\mathrm{Cu}$ concentration (Fig. 7d). There was no significant difference between NT and gNAS1 + gNAAT or gIDS3 lines in Mn concentration (Fig. 7c). In seeds from gNAS1 plants, the Mn concentration was somewhat lower than that of NT plants.

Metal concentrations of aboveground parts (leaves and stems)

To determine whether the $\mathrm{Fe}$ and $\mathrm{Zn}$ concentrations of leaves and stems was also increased, we measured the metal concentrations of aboveground structures other than the ears (i.e., leaves and stems) at harvest (Fig. 8). The average $\mathrm{Fe}$ concentration in the leaves and stems of NT, gNAS1, gNAS1+gNAAT, and gIDS3 plants was 119, 121, 145 , and $109 \mu \mathrm{g} \mathrm{g}^{-1}$, respectively (Fig. 8a). The average $\mathrm{Zn}$ concentration in the leaves and stems of NT, gNAS1, gNAS1+gNAAT, and gIDS3 plants was 24.6, 32.3, 30.7, and $22.0 \mu \mathrm{g} \mathrm{g}^{-1}$, respectively (Fig. 8b). There were no significant differences between NT and transgenic plants in $\mathrm{Fe}$ or $\mathrm{Zn}$ concentration or in the $\mathrm{Mn}$ and $\mathrm{Cu}$ concentrations in leaves and stems.

The distribution of $\mathrm{Fe}$ and $\mathrm{Zn}$ among the plant parts was measured at harvest in NT plants (Fig. 9). Fe was predominantly distributed in leaves and stems, with much less accumulation in ears and grains (Fig. 9a). The $\mathrm{Zn}$ in rice grains was relatively high compared to $\mathrm{Fe}$, but $\mathrm{Zn}$ also accumulated more in leaves and stems than in ears and grains (Fig. 9b).

\section{Discussion}

In our field trial on Andosol soils, the gIDS3 line accumulated more $\mathrm{Fe}$ and $\mathrm{Zn}$ in both brown and polished rice grains than did the NT (Figs. 6, 7). In a previous year, trial (2005), we obtained similar results in which the $\mathrm{Fe}$ and $\mathrm{Zn}$ concentrations in brown rice grains from the gIDS3 line of a previous generation were 1.21 and 1.08 times higher than those from NT grains, when they were cultivated in the same paddy field (data not shown). The Fe and $\mathrm{Zn}$ concentrations in brown rice grains from the gIDS3 line were 1.26 and 1.37 times higher than those from NT grains, when they were cultivated in the Fe-limited paddy field with calcareous soils [20]. These results also confirm that IDS3 gene would have consistent performance to increase the content of $\mathrm{Fe}$ and $\mathrm{Zn}$ in rice grains.

There are many derivatives of MAs, which serve as chelators of $\mathrm{Fe}$ and other metals (Fig. 1). Rice produces only DMA because it lacks two dioxygenase genes IDS2 and IDS3 [16]. The gIDS3 line, which had an introduced barley IDS3 gene (Fig. 2), produced both DMA and MA 
Fig. 6 Metal concentration of brown rice. $\mathrm{Fe}$ (a), $\mathrm{Zn}(\mathbf{b}), \mathrm{Mn}$ (c), and $\mathrm{Cu}(\mathbf{d})$ concentrations of brown seeds. The data represent the mean \pm standard error of four replications for each line. Different letters above the bars indicate significant differences
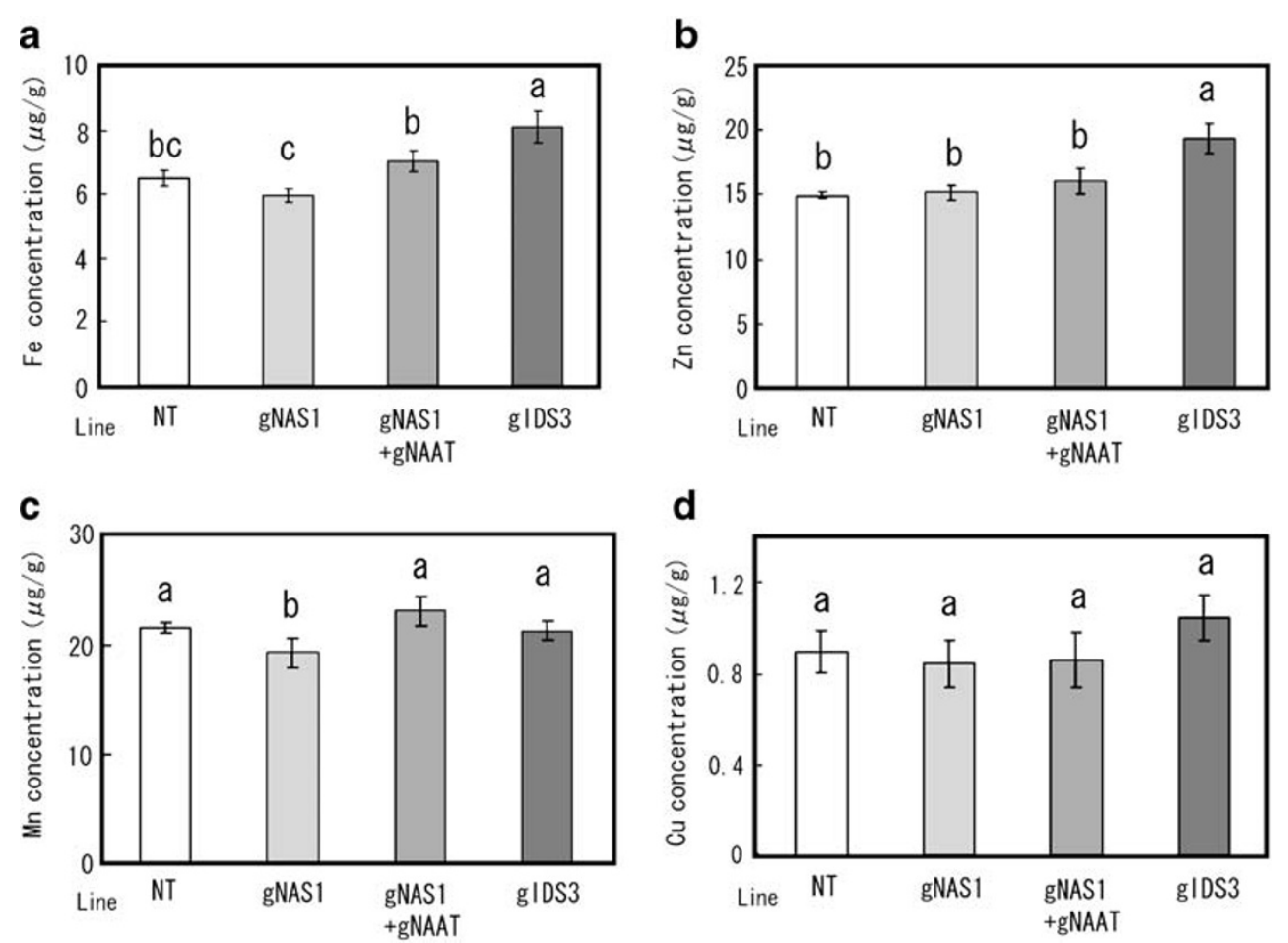

d

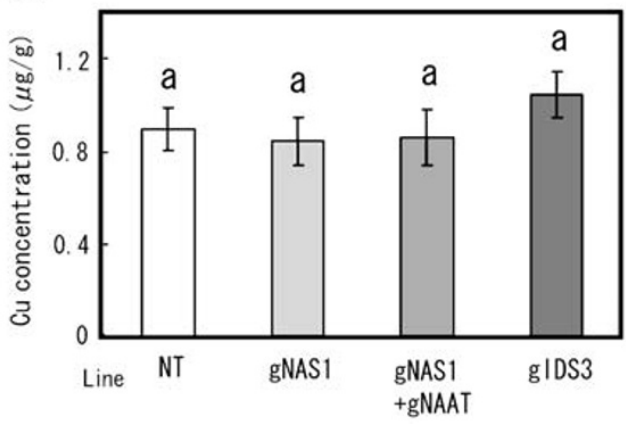

under both Fe-deficient and Fe-sufficient conditions [11]. The MA produced by IDS3 probably enhanced Fe uptake from the soil and $\mathrm{Fe}$ translocation via the xylem via the following mechanisms. The histochemical localization of $I D S 3$ promoter activity in rice indicates that IDS3 is expressed in restricted parts of the exodermis and vascular bundles of roots under Fe-sufficient conditions. Particularly strong expression can be detected within the pericycle cells that are adjacent to the protoxylem [12]. Thus, MA produced in the root exodermis of gIDS3 plants might be
Fig. 7 Metal concentration of polished rice. $\mathrm{Fe}(\mathbf{a}), \mathrm{Zn}(\mathbf{b}), \mathrm{Mn}$ (c), and $\mathrm{Cu}(\mathbf{d})$ concentrations of polished seeds. The data represent the mean \pm standard error of four replications for each line. Different letters above the bars indicate significant differences

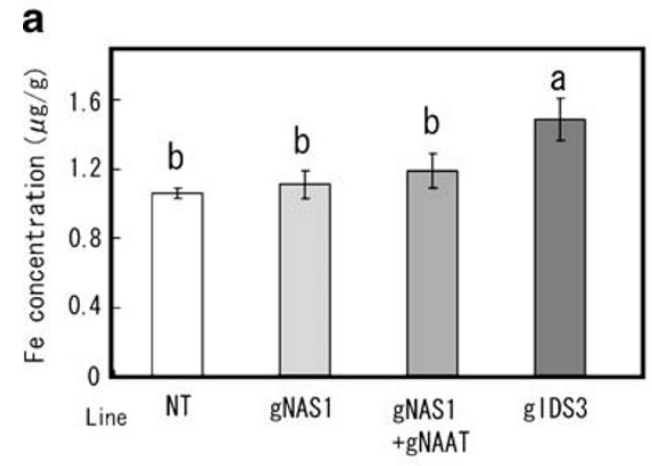

b

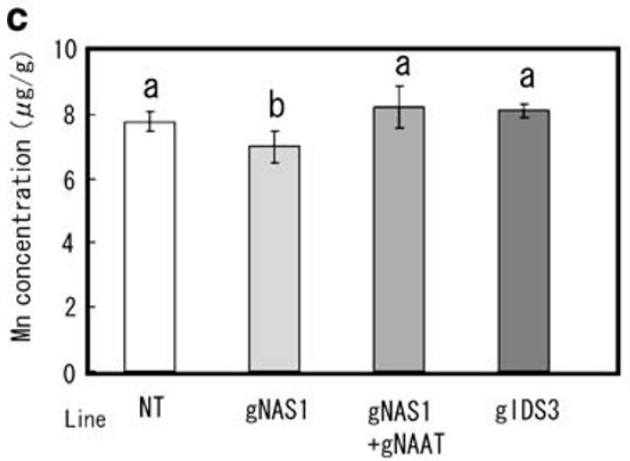

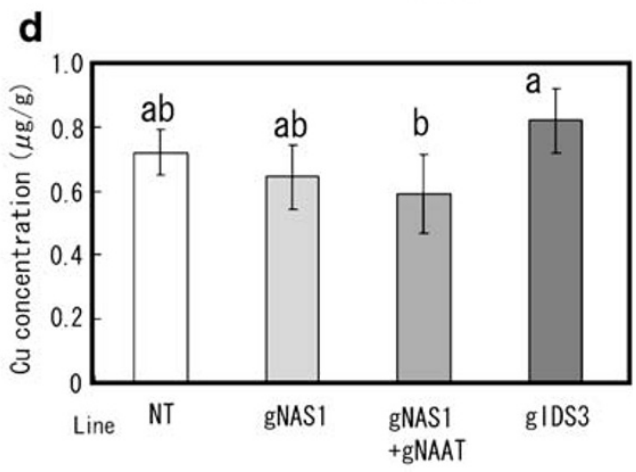


Fig. 8 Metal concentration of aboveground plant parts (leaves and stems). $\mathrm{Fe}$ (a), $\mathrm{Zn}$ (b), Mn (c), and $\mathrm{Cu}(\mathbf{d})$ concentrations of rice leaves and stems after harvest. The data represent the mean \pm standard error of four replications for each line.

Different letters above the bars indicate significant differences
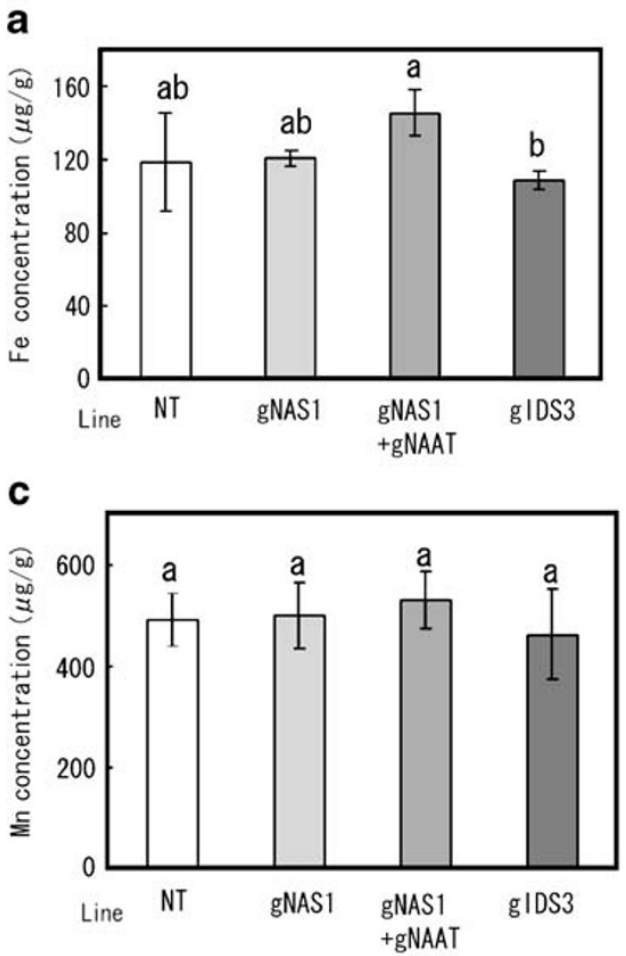
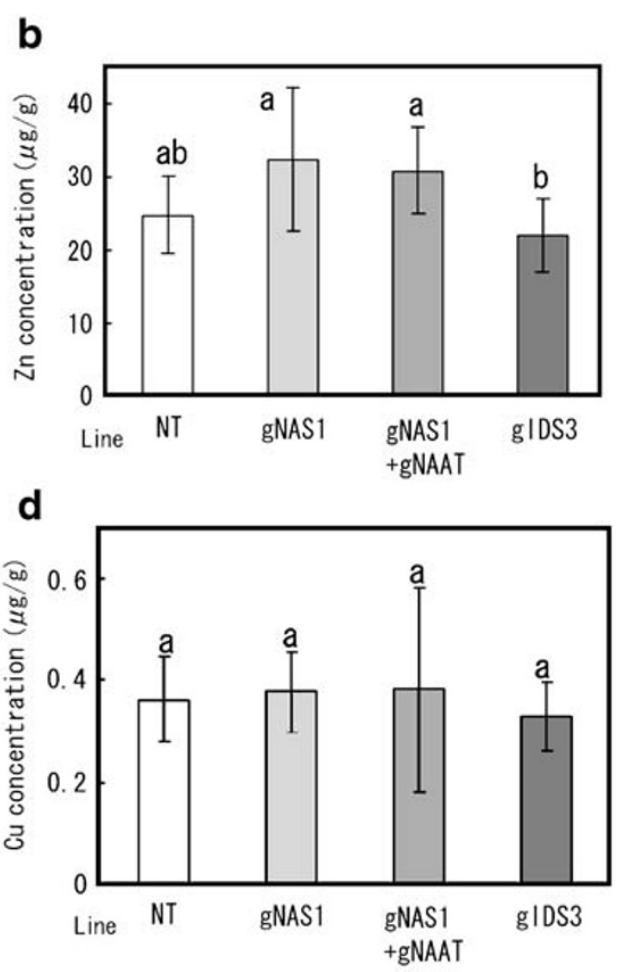

secreted into the soil and enhance Fe uptake. The MA produced in pericycle cells might then enhance $\mathrm{Fe}$ transport from roots to shoots via the xylem. However, IDS3 expression is not observed in Fe-sufficient leaves [12].

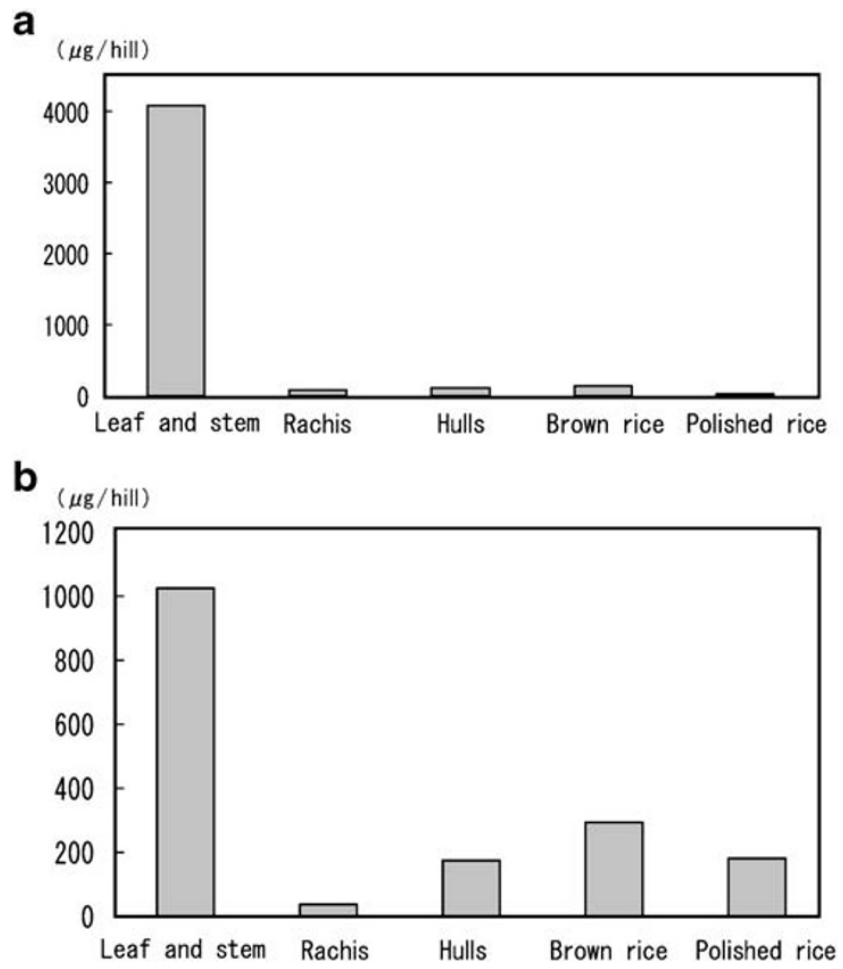

Fig. 9 Total metal content in each part of non-transgenic (NT) plants. Total Fe (a) and total Zn (b) in each part of NT plants per hill (three plants) von Wirén et al. [27] proposed that the hydroxylation of the phytosiderophore skeleton enhances the stability of MAs-Fe(III) complexes, particularly under mildly acidic conditions ( $\mathrm{pH}$ 5.0-6.5). The Andosol in which we cultivated the rice was approximately $\mathrm{pH}$ 6.0. Additionally, the rice xylem sap is pH 6.5 [15]. Thus, MA-Fe(III) complexes might be more stable than DMA-Fe(III) complexes in the Andosol soils and in rice xylem sap and would be more efficient for Fe uptake from the soil and transport within the plant. Additionally, the plasma membrane is locally acidified, and MA-Fe(III) is more stable than DMA-Fe(III) when transported through a plasma membrane [27]; thus, MA-Fe(III) might be more easily transported by a membrane transporter.

Under Fe-sufficient conditions, rice genes involved in MAs biosynthesis, including OsNAS1, OsNAS2, OsNAAT1, and $O s D M A S 1$, are expressed in the portions of roots that are involved in long-distance transport [2, 9, 10]. Thus, DMA might help to transport Fe over long distances in Fesufficient conditions. ${ }^{52} \mathrm{Fe}-\mathrm{DMA}$ applied to the roots of non-transgenic rice in the maturing stage is transported simultaneously to the ear and to the flag leaf within $4 \mathrm{~h}$ [26]. MA would also contribute to long-distance $\mathrm{Fe}$ transport in Fe-sufficient conditions, like DMA, because of its greater stability in xylem sup. Thus, transgenic lines possessing IDS3 might have enhanced Fe uptake from the soil and enhanced long-distance Fe transport via the xylem because of the production of MA. With regard to translocation via phloem, rice phloem sap is $\mathrm{pH}$ 7.8-8.0. At this $\mathrm{pH}, \mathrm{MA}-\mathrm{Fe}$ (II) complexes might be more stable than DMA$\mathrm{Fe}(\mathrm{III})$ or MA-Fe(III) complexes [27]. 
Interestingly, the $\mathrm{Zn}$ concentration was also higher in gIDS3 seeds than in the seeds of NT and other transgenic lines. DMA is involved in the long-distance transport of $\mathrm{Zn}$ in $\mathrm{Zn-deficient} \mathrm{barley} \mathrm{and} \mathrm{rice}[21,22]$. In addition, the structure of the MAs-Zn(II) complex is very similar to that of MAs-Fe(III) [27]. Although little is known about the possible effect of DMA hydroxylation on $\mathrm{Zn}$ transport, MA might have enhanced $\mathrm{Zn}$ transport into seeds in the gIDS3 line.

The $\mathrm{Fe}$ and $\mathrm{Zn}$ concentrations in leaves and stems at harvest were not increased by the introduction of IDS3 (Fig. 8a, b). Total Fe and total $\mathrm{Zn}$ in leaves and stems were much higher than those in grains (Fig. 9a, b). Thus, the effect of gIDS3 on $\mathrm{Fe}$ and $\mathrm{Zn}$ concentrations appears to be manifested in grains (Figs. 6 and 7) but not in leaves and stems (Fig. 8).

The $\mathrm{Fe}$ and $\mathrm{Zn}$ concentrations of grains did not significantly increase in the gNAS1 or gNAS $1+$ gNAAT line compared to the NT line. The transgenic rice that possesses $H v N A S 1$ genome fragment produced more nicotianamine (NA) and DMA in Fe-sufficient condition [7]. The activity of NAAT in the transgenic rice containing $H v N A A T-A$ and HvNAAT-B genome fragment was higher than that of wild type in Fe-sufficient condition [24]. Therefore, gNAS1 or gNAS1+gNAAT line would produce more NA and DMA than NT line, though they would not produce MA because of the lack of IDS3. On the other hand, gIDS3 line produced MA in Fe-sufficient condition [11]. The ability to produce MA might be the key to increasing $\mathrm{Fe}$ and $\mathrm{Zn}$ concentrations in seeds. The introduction of HvNASI, HvNAAT-A and HvNAAT-B, and IDS3 into rice in combination will enhance the $\mathrm{Fe}$ and $\mathrm{Zn}$ concentrations of the grains.

Recently, Cheng et al. [3] reported that OSNAAT1 knockout mutant rice accumulated more $\mathrm{Fe}$ in grains than WT. However, this result is not due to suppression of OSNAAT1 gene itself but is thought to be due to the response of induced Fe deficiency by OsNAAT1 suppression. In their report, OSNAAT1 knockout mutant exhibited a kind of Fe deficiency symptoms and increased expression of various genes in relation to $\mathrm{Fe}$ absorption or accumulation such as OsNAS1, OSIRT1, or OSYSL2. Expression of these genes might enhance $\mathrm{Fe}$ concentration in the grains. NA content in shoots and roots of OSNAAT1 knockout mutant was greatly increased than in WT. This higher NA content might also enhance Fe concentration in the grains, since NA serves as a general metal chelator within plants.

Previously, the main way to improve the Fe concentration of rice grains was to increase the expression of ferritin using endosperm-specific promoters [13]. We have demonstrated that the use of genes for MAs synthesis will further improve the $\mathrm{Fe}$ concentration of rice seeds. The $\mathrm{Mn}$ and $\mathrm{Cu}$ concentrations in gIDS3 grains were not increased. Because high $\mathrm{Mn}$ and $\mathrm{Cu}$ concentrations in grains are undesirable for biofortified crops, this phenotype will be very useful for mineral biofortification.

Another benefit of transgenic rice lines with enhanced MAs synthesis is that they will exhibit Fe-deficiency tolerance when grown in alkaline soils, leading to increased mineral concentrations in seeds. The gIDS3 lines that were cultivated in the paddy fields of calcareous soils showed Fe-deficiency tolerance [20]. Thus, our strategy provides an efficient method for the mineral biofortification of rice using a transgenic approach.

\section{Methods}

Plant materials

Japonica rice (Oryza sativa L.) cultivar Tsukinohikari was used as the NT control and for transformation. The transgenic rice line gNAS1 (Fig. 2a) possesses a 13.0-kb $H v N A S 1$ barley genome fragment and originated from line 24 of Higuchi et al. [8]. The transgenic rice line gIDS3 (Fig. 2c) possesses a 20-kb IDS3 barley genome fragment and originated from line 1 of Kobayashi et al. [11].

To produce the transgenic rice line gNAS1+gNAAT (Fig. 2b), a 7.6-kb barley HvNAS1 genome fragment [8] was excised using HindIII and inserted into the HindIII site of the vector pBIGRZ [1]. Then, an 11.0-kb HvNAAT-A and $H v N A A T-B$ genome fragment [25] was excised using NotI and inserted into the NotI site of pBIGRZ-NAS. The resultant vector was introduced into rice cultivar Tsukinohikari following the methods of Higuchi et al. [8].

Genomic insertion was confirmed using a polymerase chain reaction-based method and Southern blots. Gene expression was also confirmed using Northern blots under Fe-deficient conditions $[8,11]$ (data not shown).

\section{Seedling preparation}

The seeds of the three transgenic rice lines were harvested in an environmentally isolated paddy field that had Andosol soils at Tohoku University, Japan, in 2005. The seeds were sown on nursery beds for the experiment. The seeds were size-selected by soaking in $\mathrm{NaCl}$ solution. The specific gravity of the $\mathrm{NaCl}$ solution was $1.13 \mathrm{~g} / \mathrm{cm}^{3}$ for the selection of NT, gNAS1, and gNAS1+gNAAT seeds. Because there were not enough seeds from the gIDS3 line, gIDS3 seeds were selected using $1.06 \mathrm{~g} / \mathrm{cm}^{3} \mathrm{NaCl}$ solution. After size selection, the seeds were soaked overnight in fungicide solution (Momi-guard, Hokuko, Japan) and then soaked in water at $20^{\circ} \mathrm{C}$ for 6 days to break dormancy. The seeds were then incubated in water at $30^{\circ} \mathrm{C}$ overnight to accelerate germination. The seeds were sown in nursery bed 
soil by 3 April 2006. Previously germinated seeds were sown on brown soil (pH 5.5; Inaho Kumiai Muhiryou Baido, Agricultural Corporation, Japan) in small pots ( $3 \mathrm{ml})$ and then covered with the same soil. Three seeds were sown per pot, covered with soil, and grown for 45 days in a greenhouse at the environmentally isolated field.

Experimental design and transplantation

The paddy field was established at the Field Science Center of Tohoku University, Miyagi, Japan $\left(39^{\circ} \mathrm{N} ; 141^{\circ} \mathrm{E}\right)$. A $6.6 \times 6.9 \mathrm{~m}$ paddy field was used for the experiment (Figs. $3 \mathrm{a}$ and 4). The soil type was Andosol with approximate $\mathrm{pH}$ of 6.0 .

Seedlings were transplanted into the paddy field on 18 May 2006. A commercial fertilizer (Toruzou-kun; NPK= 14:20:14; Zennou, Japan) was applied at transplanting at $60 \mathrm{~kg} \mathrm{~N} \mathrm{ha}{ }^{-1}$. Each hill consisted of three plants. Column width of hill to hill was 30 and $15 \mathrm{~cm}$ (Fig. 3b). Each replication comprised eight $(120 \mathrm{~cm}) \times$ four $(120 \mathrm{~cm})$ hills (Fig. 3b). Each line was planted in four replications in the experimental area (Fig. 3a). To adjust the light and nutrient conditions between center and border replications of experimental area, NT plants were grown around the experimental area (Fig. 3a, gray zone). The paddy field was submerged in water until it was drained on 9 October 2006.

Measurement of plant growth and yield

The height of plants, number of tillers, and leaf color (SPAD value; Konika Minolta, Japan) of the largest leaves were measured for all plants in the inner six hills of each replication (Fig. 3b, black hills) on 3 June (16 DAT), 17 June (30 DAT), 29 June (42 DAT), 12 July (55 DAT), 26 July (69 DAT), and 27 August (101 DAT). Plants headed at around 23 August (97 DAT). The plants were harvested on 23 October (158 DAT). After harvest, the total number of seeds and grain weight for the inner six hills from each replication were measured to determine the grain yield.

Metal concentration analyses

Brown rice grains (specific gravity $>1.06 \mathrm{~g} / \mathrm{cm}^{3}$ ) were collected randomly from all plants from the inner six hills of each replication (Fig. 3b, black hills) for metal concentration analyses. Ten seeds were digested with $1 \mathrm{ml}$ of $13 \mathrm{M} \mathrm{HNO}_{3}$ and $1 \mathrm{ml}$ of $8.8 \mathrm{M} \mathrm{H}_{2} \mathrm{O}_{2}$ (Wako, Japan) at $200^{\circ} \mathrm{C}$ for $20 \mathrm{~min}$ using MARS Xpress (CEM, USA); seeds were digested in six replicate subsamples from each replication. After digestion, the samples were collected and diluted to $5 \mathrm{ml}$ and analyzed using a SPS1200VR ICPAES (Seiko, Japan).
To obtain polished seeds, 60 brown seeds were placed into a 2-ml tube and shaken at 2,500 rpm for $150 \mathrm{~s}$, at least four times, using a Multi-beads Shocker (Yasui Kikai, Japan). Approximately 25 well-polished seeds from each replication were digested and their metal concentration measured.

The aboveground parts other than ears (i.e., leaves and stems) were harvested. The plant materials were ground using a crushing machine (Fujiwara Seisakusho, Japan). Approximately 250-300 $\mathrm{mg}$ of the ground powder was digested and the metal concentration was measured.

Approximately $200 \mathrm{mg}$ each of rachis and hulls were collected from each replication of NT plants, digested, and the metal concentration was measured. The total metal concentration of each plant part (i.e., leaves and stems, rachis, hulls, brown rice, and polished rice) was calculated.

\section{Statistics}

Data were compared using analysis of variance or a Student-Newman-Keuls test; $P<0.05$ was considered statistically significant.

Acknowledgments We thank R. Yamamoto, Dr. S. Nagasaka, Dr. Y. Ishimaru, Dr. T. Nozoye, Y. Ogo, Y. Kakei, I. Ogawa, K. Usuda, R. N. Itai, S. Hugo, Dr. K. Bashir, Dr. T. Tsukamoto, and T. Aoyama for their technical assistance during the field trial. We received permission of field research using transgenic rice varieties from Ministry of Agriculture, Foresty and Fisheries of Japan and State Minister for the Environment of Japan on 25 April 2005.

Open Access This article is distributed under the terms of the Creative Commons Attribution Noncommercial License which permits any noncommercial use, distribution, and reproduction in any medium, provided the original author(s) and source are credited.

\section{References}

1. Akiyama K, Nakamura S, Suzuki T, Wisniewska I, Sasaki N, Kawasaki S. Development of a system of rice transformation with long genome inserts for their functional analysis for positional cloning. Plant Cell Physiol 1997;38:s94.

2. Bashir K, Inoue H, Nagasaka S, Takahashi M, Nakanishi H, Mori $\mathrm{S}$, Nishizawa NK. Cloning and characterization of deoxymugineic acid synthase genes from graminaceous plants. J Biol Chem 2006;281:32395-402.

3. Cheng L, Wang F, Shou H, Huang F, Zheng L, He F, Li J, Zhao FJ, Ueno D, Ma JF, Wu P. Mutation in nicotianamine aminotransferase stimulated the $\mathrm{Fe}(\mathrm{II})$ acquisition system and led to iron accumulation in rice. Plant Physiol 2007;145:1647-57.

4. Curie C, Panaviene Z, Loulergue C, Dellaporta SL, Briat JF, Walker EL. Maize yellow stripel encodes a membrane protein directly involved in Fe(III) uptake. Nature 2001;409:346-9.

5. Goto F, Yoshihara T, Shigemoto N, Toki S, Takaiwa F. Iron fortification of rice seed by the soybean ferritin gene. Nature Biotechnol 1999;17:282-6.

6. Higuchi K, Suzuki K, Nakanishi H, Yamaguchi H, Nishizawa NK, Mori S. Cloning of nicotianamine synthase genes, novel genes 
involved in the biosynthesis of phytosiderophores. Plant Physiol 1999;119:471-9.

7. Higuchi K, Takahashi M, Nakanishi H, Kawasaki S, Nishizawa NK, Mori S. Analysis of transgenic rice containing barley nicotianamine synthase gene. Soil Sci Plant Nutr 2001;47:315-22.

8. Higuchi K, Watanabe S, Takahashi M, Kawasaki S, Nakanishi H, Nishizawa NK, Mori S. Nicotianamine synthase gene expression differs in barley and rice under Fe-deficient conditions. Plant $\mathrm{J}$ 2001;25:159-67.

9. Inoue $\mathrm{H}$, Higuchi $\mathrm{K}$, Takahashi $\mathrm{M}$, Nakanishi $\mathrm{H}$, Mori $\mathrm{S}$, Nishizawa NK. Three rice nicotianamine synthase genes, OsNAS1, OsNAS2, and OsNAS3 are expressed in cells involved in long-distance transport of iron and differentially regulated by iron. Plant J 2003;36:366-81.

10. Inoue H, Takahashi M, Kobayashi T, Suzuki M, Nakanishi H, Mori S, Nishizawa NK. Identification and localisation of the rice nicotianamine aminotransferase gene $O S N A A T 1$ expression suggests the site of phytosiderophore synthesis in rice. Plant Mol Biol 2008;66:193-203.

11. Kobayashi T, Nakanishi H, Takahashi M, Kawasaki S, Nishizawa $\mathrm{NK}$, Mori S. In vivo evidence that Ids 3 from Hordeum vulgare encodes a dioxygenase that converts 2 '-deoxymugineic acid to mugineic acid in transgenic rice. Planta 2001;212:864-71.

12. Kobayashi T, Nakayama Y, Takahashi M, Inoue H, Nakanishi H, Yoshihara T, Mori S, Nishizawa NK. Construction of artificial promoters highly responsive to iron deficiency. Soil Sci Plant Nutr 2004;50:1167-75.

13. Lucca P, Poletti S, Sautter C. Genetic engineering approaches to enrich rice with iron and vitamin A. Physiol Plant 2006;126:291303.

14. Mihashi S, Mori S. Characterization of mugineic acid-Fe transporter in Fe-deficient barley roots using the multicompartment transport box method. Biol Metals 1989;2:146-54.

15. Mitani N, Ma JF, Iwashita T. Identification of the silicon form in xylem sap of rice (Oryza sativa L.). Plant Cell Physiol 2005;46:279-83.

16. Nakanishi H, Yamaguchi H, Sasakuma T, Nishizawa NK, Mori S. Two dioxygenase genes, Ids3 and Ids2, from Hordeum vulgare are involved in the biosynthesis of mugineic acid family phytosiderophores. Plant Mol Biol 2000;44:199-207.
17. Qu LQ, Yoshihara T, Ooyama A, Goto F, Takaiwa F. Iron accumulation does not parallel the high expression level of ferritin in transgenic rice seeds. Planta 2005;222:225-33.

18. Sandstead HH. Zinc deficiency: a public health problem? Am J Dis Child 1991;145:853-9.

19. Stoltzfus RJ, Dreyfuss ML. Guidelines for the use of iron supplements to prevent and treat iron deficiency anemia. Washington, DC: ILSI Press; 1998.

20. Suzuki M, Morikawa KC, Nakanishi H, Takahashi M, Saigusa M, Mori S, Nishizawa NK. Transgenic rice lines that include barley genes have increased tolerance to low iron availability in a calcareous paddy soil. Soil Sci Plant Nutr 2008;54:77-85.

21. Suzuki M, Takahashi M, Tsukamoto T, Watanabe S, Matsuhashi S, Yazaki J, Kishimoto N, Kikuchi S, Nakanishi H, Mori S, Nishizawa NK. Biosynthesis and secretion of mugineic acid family phytosiderophores in zinc-deficient barley. Plant J 2006;48:85-97.

22. Suzuki M, Tsukamoto $T$, Inoue $H$, Watanabe $S$, Matsuhashi $S$, Takahashi M, Nakanishi H, Mori S, Nishizawa NK. Deoxymugineic acid increases $\mathrm{Zn}$ translocation in $\mathrm{Zn}$-deficient rice plants. Plant Mol Biol 2008;66:609-17.

23. Takagi S. Naturally occurring iron-chelating compounds in oatand rice-root washings. Soil Sci Plant Nutr 1976;22:423-33.

24. Takahashi M, Nakanishi H, Kawasaki S, Nishizawa NK, Mori S. Enhanced tolerance of rice to low iron availability in alkaline soils using barley nicotianamine aminotransferase genes. Nature Biotechnol 2001;19:466-9.

25. Takahashi M, Yamaguchi H, Nakanishi H, Shioiri T, Nishizawa NK, Mori S. Cloning two genes for nicotianamine aminotransferase, a critical enzyme in iron acquisition (Strategy II) in graminaceous plants. Plant Physiol 1999;121:947-56.

26. Tsukamoto T, Nakanishi H, Uchida H, Ogusu T, Nishiyama S, Watanabe S, Matsuhashi S, Nishizawa NK, Mori S. Real time visualization of ${ }^{52} \mathrm{Fe}$ and ${ }^{62} \mathrm{Zn}$ translocation in the ear of rice. Plant Cell Physiol 2006;47:S150.

27. von Wirén N, Khodr H, Hider CR. Hydroxylated phytosiderophore species possess an enhanced chelate stability and affinity for iron(III). Plant Physiol 2000;124:1149-57.

28. World Health Organization (WHO). World Health Report Reducing Risks, Promoting Healthy Life. WHO: Geneva, Switzerland; 2002. 